\author{
Military Technical \\ College \\ Kobry El-Kobba \\ Cairo, Egypt
}

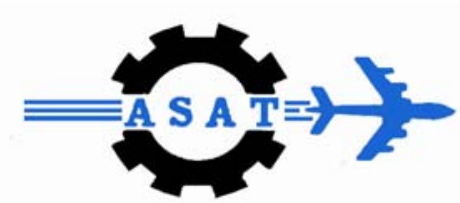

12-th International

Conference on

Aerospace Sciences \&

Aviation Technology

\title{
FREE FLYER MANOEUVERING ROUND A SPACE STATION
}

\author{
BADAWY A. * and MCINNES C.R..*
}

\begin{abstract}
One of the most critical processes of a free-flying autonomous robot is investigated in this paper using artificial potential fields. Close navigation round The International Space Station could not be established with the potential functions without representing the station using a superquadric model. The final configuration is defined as the global minimum of a function which includes the goal parameters and the station structure. An error quaternion representation is used to define both attractive and repulsive potentials to enable the formulation of a position-orientation dependent controller. Coupling between translational and rotational motions leads to better controller performance. Its elegancy and simplicity minimize the computational power needed for the free-flyer on-board computer.
\end{abstract}

\section{KEY WORDS}

Space robotics, ISS, superquadric, potential field method, quaternions, motion planning.

\footnotetext{
* Graduate student, Dpt. of Mech. Eng., University of Strathclyde, Glasgow, UK ** Professor, Dpt. of Mech. Eng., University of Strathclyde, Glasgow, UK
} 


\section{NOMENCLATURE}

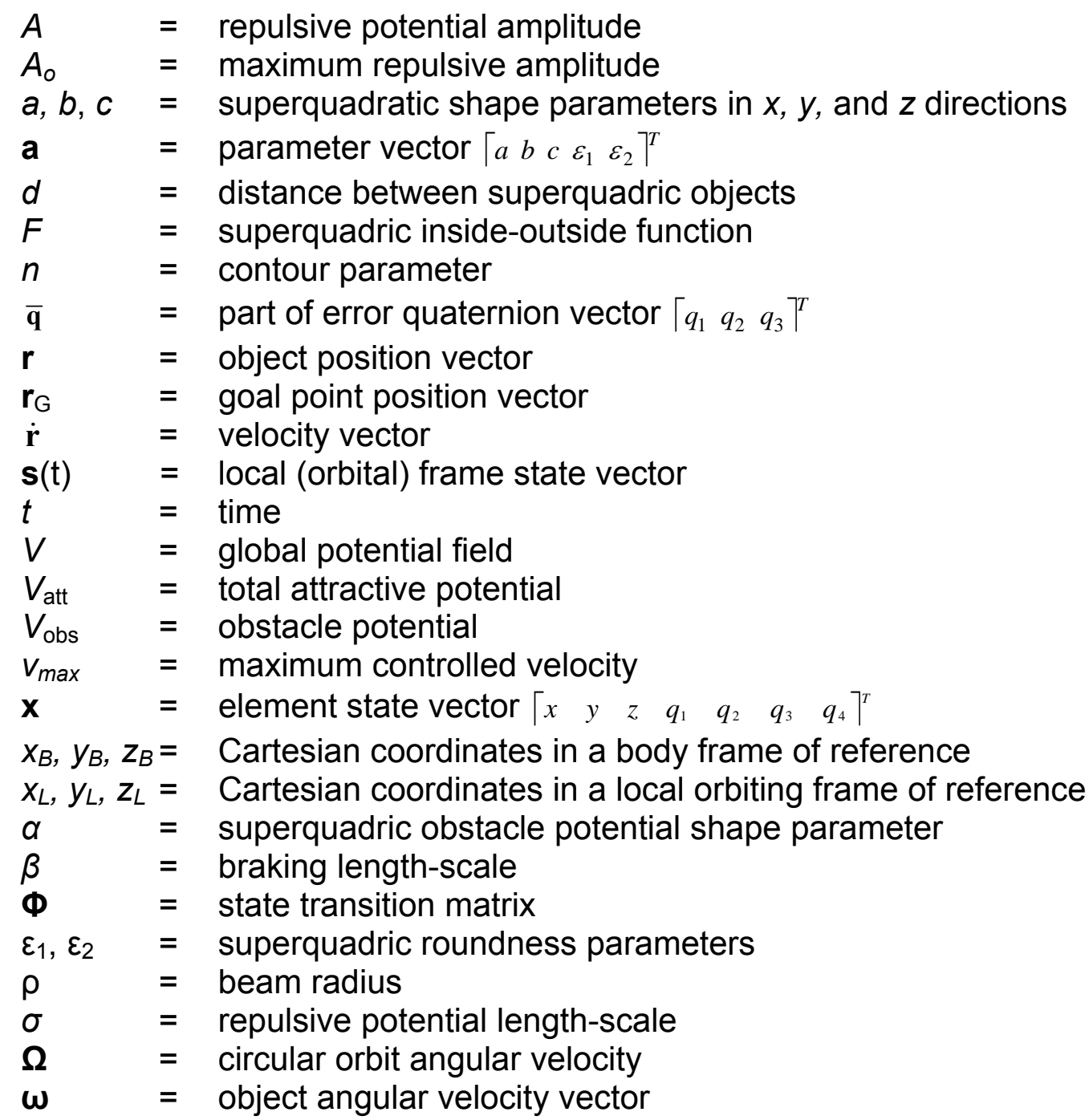

\section{INTRODUCTION}

Free flying robots enable flexible assembly and service facilities to work inside space facilities or in the free space. They serve in conjunction with space redundant manipulators and astronauts with the advantage of flexibility over the first and safety over the second type.

Formation of a potential field incorporating both translational and rotational motion is used herein to define motion parameters of a free-flyer maneuvering at the International Space Station. The global potential is composed of attractive and repulsive potentials. Attractive potentials bring the free-flyer toward its goal configuration, while repulsive ones protect it and the station structure from collisions[1]. 
Wide Space applications for the potential field method include proximity manoeuvring [2], large angle slew manoeuvres [3], and formation-flying [4]. On-orbit assembly of large structures is another major application of the method [5-7].

Distinctive station modules using the same model, nevertheless their shape and size differences are defined using superquadric functions. They are formed in local body frames of reference; hence error quaternions are used to find relations between local and inertial parameters. These functions are modified to deform from the modules shapes to spheres and combined with attractive potentials [8,9]. The deformation rate is controlled through the relative distance to avoid local minima formation due to overlap of multiple objects interface.

The repulsive potentials depend on the relative distance between the free-flyer and the ISS modules. As they have different sizes and orientations, a rigid body formulation is required instead of considering only their centers to calculate the separation distances [10].

The potential function proposed in this paper is aimed to minimize on-board sensors requirements through using only kinematic data as the measured quantities. The required impulses are calculated on-board, then produced by small thrusters, whereas a continuous control torque is produced from control moment gyros.

\section{ATTRACTIVE POTENTIAL}

A parabolic attractive well is chosen to drive the free-flyer toward its goal configuration. The translational attractive field is a function of the Euclidian distance between the free-flyer and its goal position, Fig. 1 . The error quaternions, $\overline{\mathbf{q}}$ which are the difference between the quaternion of the free-flyer and its goal orientation, represent the rotational attractive field as:

$$
V_{a t t}=\frac{\lambda_{p}}{2}\left(\mathbf{r}-\mathbf{r}_{G}\right) \cdot\left(\mathbf{r}-\mathbf{r}_{G}\right)+\frac{\lambda_{q}}{2} \overline{\mathbf{q}} \cdot \overline{\mathbf{q}}
$$

The free-flyer will move down the gradient of the potential field towards this global minimum where its position vector $r$ equals the goal position vector $r_{G}$. The first three terms in the error quaternion vector, $\mathbf{q}=\left\lceil\begin{array}{llll}q_{1} & q_{2} & q_{3} & q_{4}\end{array}\right]^{T}$, are set to zero at the goal orientation. The two constants $\lambda_{p}$ and $\lambda q$ represent the ratio of the translation to rotation capability of the free-flyer.

\section{SUPERQUADRIC FUNCTION}

Superquadrics are mathematical representations of solid objects. They are a set of parametric functions that have great utility in object modeling. Their parametric characteristics enable the creation of a range of object shapes by manipulating the so-called roundness and shape parameters as demonstrated in Fig. 2. A generic 
implicit superquadric function (inside-outside function) is defined in body axes as $[11,12]$ :

$$
F\left(\mathbf{a}, \mathbf{x}_{B}\right)=\left[\left(\frac{x_{B}}{a}\right)^{\frac{2}{\varepsilon_{2}}}+\left(\frac{y_{B}}{b}\right)^{\frac{2}{\varepsilon_{2}}}\right]^{\frac{\varepsilon_{2}}{\varepsilon_{1}}}+\left(\frac{z_{B}}{c}\right)^{\frac{2}{\varepsilon_{1}}}
$$

The superquadric surface satisfies the equation:

$$
F\left(\mathbf{a}, \mathbf{x}_{B}\right)=1
$$

where the vector $a$, represents superquadric radii $a, b$, and $c$ and the roundness parameters $\epsilon_{1}$, and $\epsilon_{2}$. The vector $\mathbf{x}_{B}$ is the position vector w.r.t. a body frame of reference. The approximate minimum distance between two superquadric solid models (object and obstacle) is found to be [10]:

$$
d\left(\mathbf{a}_{o b s}, \mathbf{a}_{o b j}, \mathbf{x}_{o b s, B}, \mathbf{x}_{o b j, B}\right)=\mid \mathbf{r}_{o b j / o b s}\left[1-F\left(\mathbf{a}_{o b s}, \mathbf{x}_{o b s, B}\right)^{\frac{-\varepsilon_{1, o b s}}{2}}-F_{o b j}\left(\mathbf{a}_{o b j}, \mathbf{x}_{o b j, B}\right)^{\frac{-\varepsilon_{1, o b j}}{2}}\right]
$$

A suitable form of the superquadric function is suggested, depending on the obstacle shape, to define a deformable solid model that takes an object shape near its edges, while deforming to a sphere at some distance from them $[8,9]$.

\section{REPULSIVE POTENTIAL}

Repelling the free-flyer away from the station structure is achieved through a repulsive potential. A Superquadric repulsive potential is defined according to the separation distance between the free-flyer and station structure, $d$, as $[8,9]$ :

for $d \geq d_{\min }$

$$
V_{o b s}=A \frac{e^{-\alpha d}}{d}
$$

for $d<d_{\min }$

$$
V_{o b s}=A e^{-\alpha d^{1+\frac{1}{\alpha}}}
$$

where $d_{\min }$ is a predefined distance depends on objects under investigation, normally unity. The repulsive potential amplitude, $A$, should be set to zero at the final docking to allow smooth contact as:

$$
A=A_{o}\left(1-e^{-\left|\mathbf{r}-\mathbf{r}_{\mathbf{G}}\right|^{2} / \sigma}\right)
$$

The exponent $\alpha$, controls the rate of the superquadric shape change from the obstacle shape to a spherical one. $A_{o}$ is the maximum amplitude and $\sigma$ is the standard deviation of the Gaussian distribution [7]. 
A separation distance calculation considering the object centre is not adequate as the station modules shape, size, and orientation should be considered. Figure 3 illustrates how the object orientation affects the distance $d$.

\section{GLOBAL POTENTIAL}

Attractive and repulsive potentials are now combined to form a global field. It is used to drive the free-flyer from its initial parking configuration to its final one while avoiding collision with station modules. The overall potential field should satisfy the stability criteria defined by Lyapunov's second theorem [13]. The proposed global function is expressed as:

$$
V=\frac{\lambda_{p}}{2}\left(\mathbf{r}-\mathbf{r}_{G}\right) \cdot\left(\mathbf{r}-\mathbf{r}_{G}\right)+\frac{\lambda_{q}}{2} \overline{\mathbf{q}} \cdot \overline{\mathbf{q}}+V_{\text {obs }}
$$

The time derivative is:

$$
\dot{V}=\lambda_{p} \dot{\mathbf{r}} \cdot\left(\mathbf{r}-\mathbf{r}_{G}\right)+\lambda_{q} \dot{\overline{\mathbf{q}}} \cdot \overline{\mathbf{q}}+\nabla V_{o b s} \dot{\mathbf{r}}+\nabla^{q} V_{o b s} \cdot \dot{\overline{\mathbf{q}}}
$$

where

$$
\begin{gathered}
\nabla=\left\lceil\frac{\partial}{\partial x} \frac{\partial}{\partial y} \frac{\partial}{\partial z}\right]^{T} \\
\nabla^{q}=\left\lceil\frac{\partial}{\partial q_{1}} \frac{\partial}{\partial q_{2}} \frac{\partial}{\partial q_{3}}\right\rceil^{T}
\end{gathered}
$$

To set the time derivative of the potential function to be negative semi-definite, the control laws will be:

$$
\dot{\mathbf{r}}=-v_{\max }\left(1-e^{-\beta V_{a t t}}\right) \frac{\nabla V}{\left|\nabla^{*} V\right|}
$$

and

$$
\dot{\overline{\mathbf{q}}}=-\omega_{\max }\left(1-e^{-\beta V_{\text {att }}}\right) \frac{\nabla^{q} V}{\left|\nabla^{*} V\right|}
$$

where

$$
\nabla^{*}=\left\lceil\frac{\partial}{\partial x} \frac{\partial}{\partial y} \frac{\partial}{\partial z} \frac{\partial}{\partial q_{1}} \frac{\partial}{\partial q_{2}} \frac{\partial}{\partial q_{3}}\right\rceil^{T}
$$


The angular velocity is calculated as [14]:

$$
\boldsymbol{\omega}=2 \mathbf{Q}^{-1} \dot{\overline{\mathbf{q}}}
$$

\section{ISS SUPERQUADRIC MODEL}

An approximate model of the ISS is formed using superquadric functions, Fig. 4. Superquadric functions prove their ability to give a simple mathematical formulation of all station modules. For parallelepiped elements both $\epsilon_{1}$ and $\epsilon_{2}$ tend to zero hence, the inside-outside function for a deformable superquadric is expressed as [8]:

$$
F\left(\mathbf{a}, \mathbf{x}_{B}\right)=\left(\frac{x_{B}}{a}\right)^{2 n}+\left(\frac{b}{a}\right)^{2}\left(\frac{y_{B}}{b}\right)^{2 n}+\left(\frac{c}{a}\right)^{2}\left(\frac{z_{B}}{c}\right)^{2 n}
$$

The parameter $\mathrm{n}$ characterizes contour surfaces as $n \rightarrow \infty$ at the parallelepiped modules edges, whereas $n \rightarrow 1$ away from them as:

$$
n=\frac{1}{1-e^{-\alpha d}}
$$

For cylindrical modules of radius $\rho$ and length $c$, the inside-outside function is defined as:

$$
F\left(\mathbf{a}, \mathbf{x}_{B}\right)=\left[\left(\frac{x_{B}}{\rho}\right)^{2}+\left(\frac{y_{B}}{\rho}\right)^{2}\right]^{n}+\left(\frac{c}{\rho}\right)^{2}\left(\frac{z_{B}}{c}\right)^{2 n}
$$

\section{FREE-FLYER SIMULATION}

A free-flyer parked on the station surface will maneuver to another point to perform certain operations like inspection, installation, or repair. The free-flyer is equipped with thrusters to enable it to perform the required maneuver. Thrusters are on when the time derivative of the global potential function is larger than some non-positive value, $c_{f}$. Control actuation is then required when:

$$
\dot{V}_{i} \geq c_{f}
$$

As this rate satisfies Eq. (18), the free-flyer will move according to the orbital mechanics equations using the Clohessy-Wiltshire approximation since the relative distance between start and goal positions are much smaller than that distance to the Earth's centre. Solution of the linearised differential equations of motion lead to describe the free flyer motion with the state transition matrix $\Phi(t)$ as:

$$
\mathbf{s}(t)=\boldsymbol{\Phi}(t) \mathbf{s}(0)
$$


where $\mathbf{s}(t)=\left[\begin{array}{llllll}x_{L} & y_{L} & z_{L} & \dot{x}_{L} & \dot{y}_{L} & \dot{z}_{L}\end{array}\right]^{T}$ w.r.t. a local orbiting frame of reference, and $\mathbf{s}(0)$ is the initial conditions for the current period of free flight between impulses. The state transition matrix can then be defined as [15]:

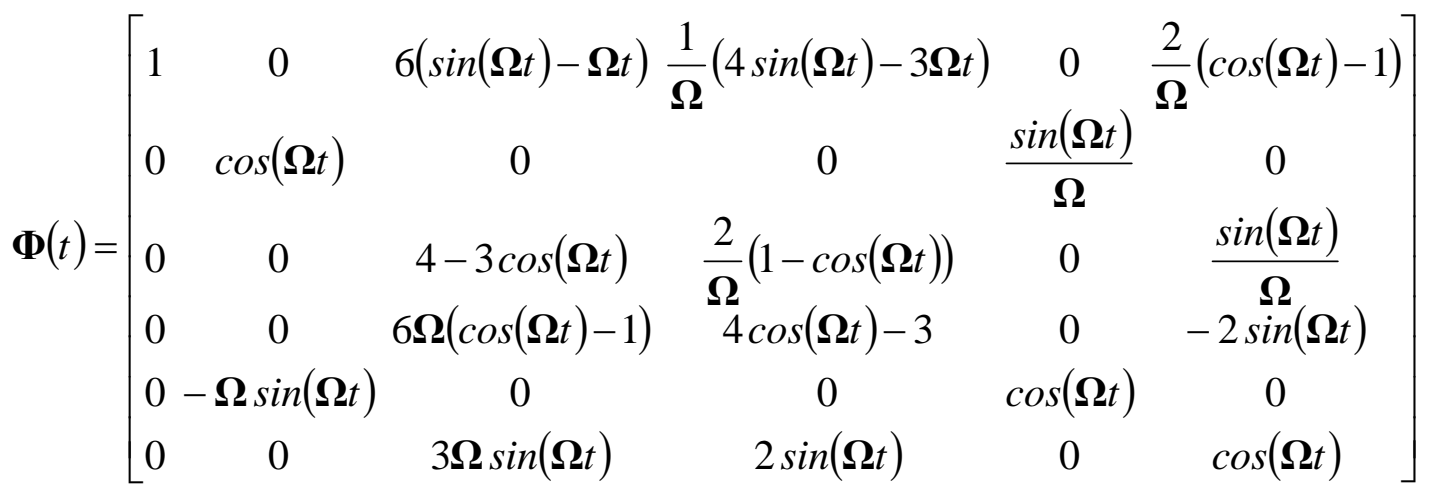

where $\boldsymbol{\Omega}$ is the natural circular angular velocity of the station orbit.

Trajectory modifications are shown at each impulse as shown in Fig. 5-a. Coupling between translation and rotation produces the quaternion change in Fig. 5-b although initial and goal orientations are identical.

The impulses required to perform the mission described in Fig. 5-a are shown in Fig.6.

\section{CONCLUSIONS}

The potential field method proved its ability to perform successful motion planning of a free-flying robot used with the ISS. Removing translation velocity and angular velocity sensors can be compensated through proper definition of the control algorithm. Merging between impulsive motion and natural orbital motion of the freeflyer was carried out considering a pure impulse by limiting the maximum change in the object velocity instead of assuming infinite power thrusters. Coupling between translational and rotational motion facilitates the free-flyer motion in case of obstacles nearby through decreasing its global potential using both motions to avoid collision whilst maintaining a continuous approach to its goal.

\section{REFERENCES}

[1] Khatib O., "Real-Time Obstacle Avoidance for Manipulators and Mobile Robots", the International Journal of Robotics Research, 1986.

[2] Roger, A.B., and Mclnnes, C.R., "Passive-Safety Constrained Free-Flyer PathPlanning with Laplace Potential Guidance at the International Space Station", Journal of Guidance, Control and Dynamics, Vol. 23, No. 6, 2000, pp. 971-979.

[3] Mclnnes C.R., "Potential Function Methods for Autonomous Spacecraft Guidance and Control", AAS 95-447, AAS/AIAA Astrodynamics Specialist Conference, Halifax, Canada, August, 1995. 
[4] McQuade, F., Ward, R., Ortix, F., and Mclnnes, C.R., "The Autonomous Configuration of Satellite Formations using Generic Potential Functions", 3rd International Workshop on Satellite Constellations and Formation-Flying, Pisa, Italy, February, 2003.

[5] McQuade, F., and Mclnnes, C.R., "Autonomous Control for On-Orbit Assembly Using Potential Function Methods", The Aeronautical Journal, Vol. 101, No. 1008, 1997, pp. 255-262.

[6] Izzo D., Pettazzi L., and Ayre M., "Mission Concept for Autonomous on Orbit Assembly of a Large Reflector in Space", IAC-05-D1.4.03, 56th International Astronautical Congress, Fukoka, Japan, September, 2005.

[7] Badawy A., and Mclnnes C.R., "Autonomous Structure Assembly Using Potential Field Functions", IAC-06-C1.P.3.04, 57th International Astronautical Congress, Valencia, Spain, October, 2006.

[8] Volpe R., "Real and Artificial Forces in the Control of Manipulators: Theory and Experiments" Ph. D thesis, 1990.

[9] Volpe R., Khosla P.," Manipulator Control with Superquadric Artificial Potential Functions: Theory and Experiments", IEEE Transaction on Systems, Man, and Cybernetics, 1990.

[10] Badawy A. and Mclnns C.R., "Separation Distance for Robot Motion Control using Superquadric Obstacle Potentials", International Control Conference, Glasgow, Scotland, 2006.

[11] Barr A. H., "Superquadrics and Angle-preserving Transformations", IEEE Computer Graphics and Applications, vol 1, pp. 11-23, 1981.

[12] Solina F., and Bajcsy R., "Recovery of Parametric Models from Range Images: The Case for Superquadrics with Global Deformations", IEEE transaction on pattern analysis and machine intelligence, vol. 12, no. 2, pp. 131-147, February 1990.

[13] Sastry S., "Nonlinear Systems", Springer-Verlag, New York Inc., 1999.

[14] Wie B., 1998 "space vehicle dynamics and control", AIAA Education series".

[15] Yu S., "On the Dynamics and Control of the Relative Motion between Two Spacecraft", Acta Astronautica, Vol. 35, No. 6, 1995, pp. 403-409. 


\section{FIGURES}

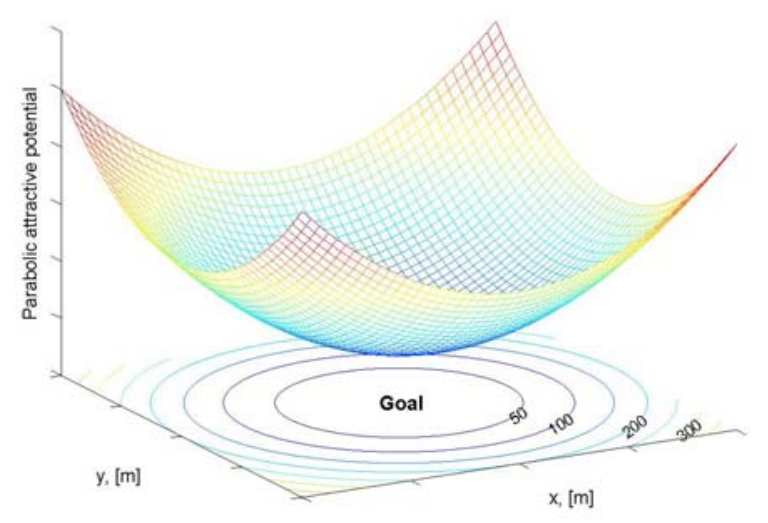

Fig. 1 Parabolic-well attractive potential
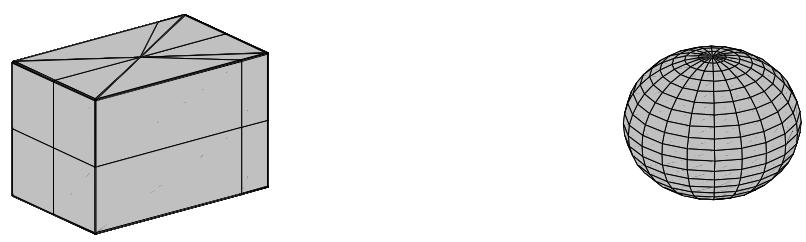

$$
\epsilon_{1}=0.01 \text {, and } \epsilon_{2}=0.01
$$

$$
\epsilon_{1}=1 \text {, and } \epsilon_{2}=1
$$
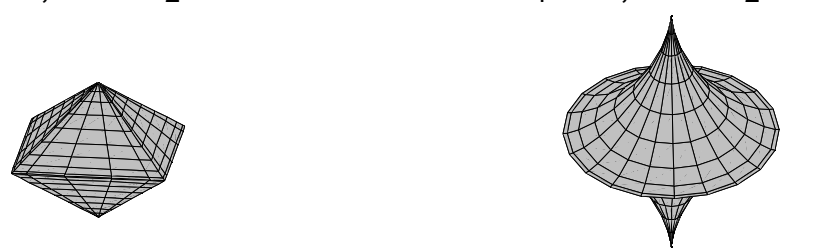

$\epsilon_{1}=2$, and $\epsilon_{2}=2$

$\epsilon_{1}=3$, and $\epsilon_{2}=1$

Fig. 2 Superquadric shapes

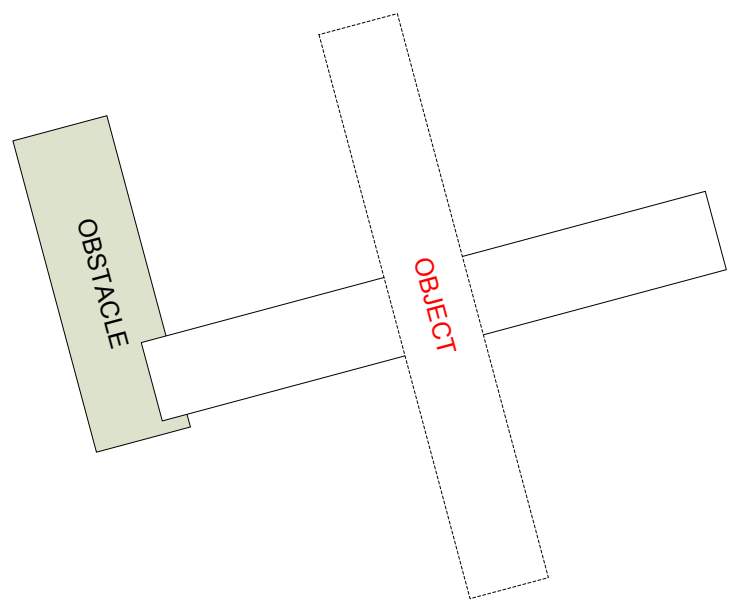

Fig. 3 Orientation effect on separation distance 


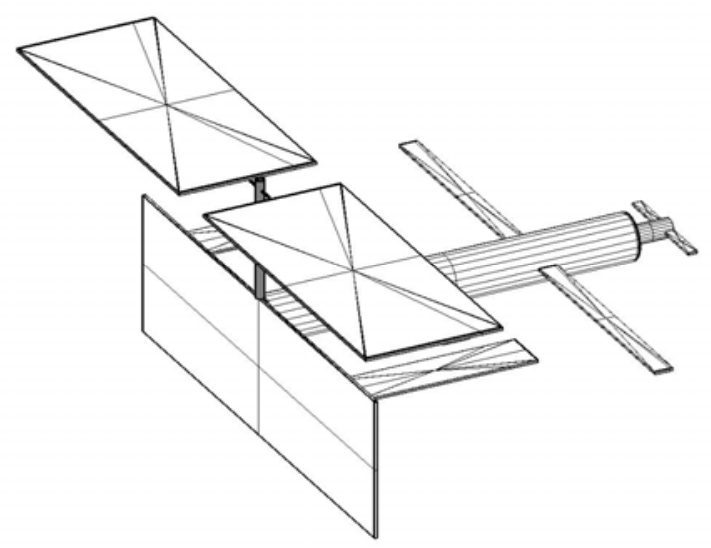

Fig. 4 International space station model

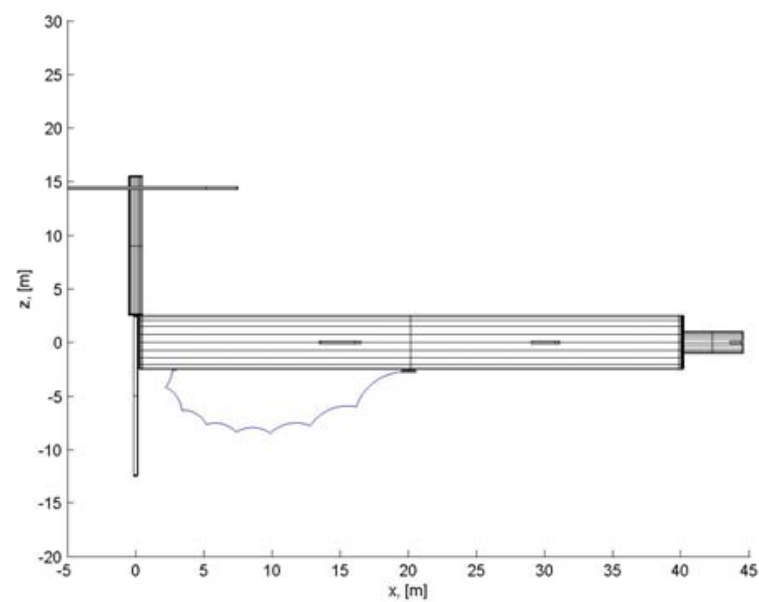

Fig. 5-a) Free-flyer trajectory

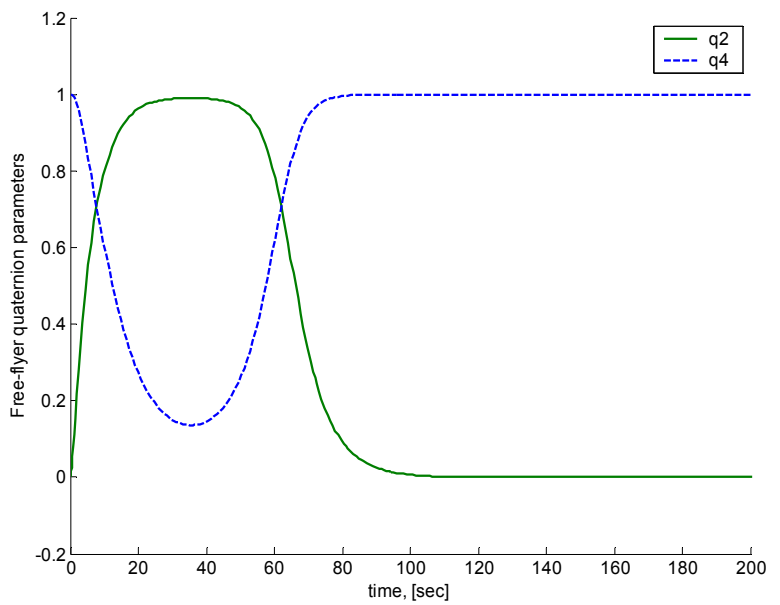

Fig. 5-b) Free-flyer rotation 


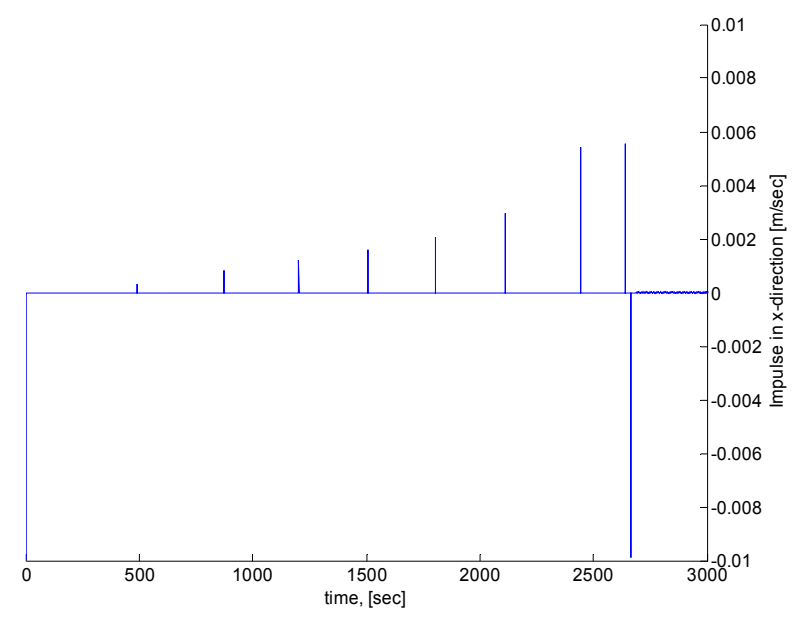

Fig. 6-a) Free-flyer thrust impulses in $x$-direction

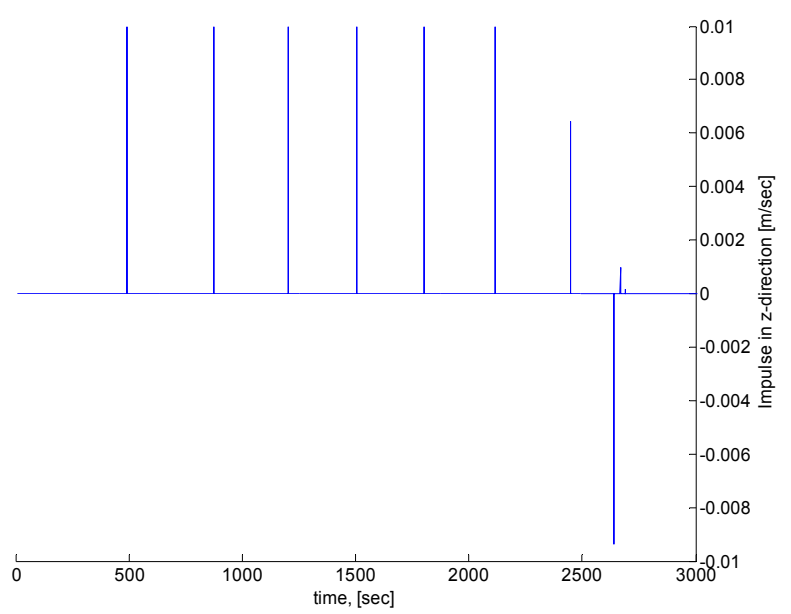

Fig. 6-b) Free-flyer thrust impulses in z-direction 